\title{
Revisão Sistemática da Utilização de Fóruns Inteligentes para a Promoção da Colaboração na Aprendizagem Virtual
}

\author{
Systematic Review of the Use of Intelligent Forums for Promoting Collaboration in Virtual \\ Learning
}

\author{
Priscilla Batista Mendes \\ Grupo SABER Tecnologias \\ Educacionais e Sociais, \\ Universidade Federal de \\ Pernambuco \\ priscy285@gmail.com
}

\author{
Josiane Lemos Machiavelli \\ Grupo SABER Tecnologias \\ Educacionais e Sociais, \\ Universidade Federal de \\ Pernambuco \\ josiane.machiavelli@gmail.com
}

\author{
Cristine Martins Gomes de Gusmão \\ Grupo SABER Tecnologias \\ Educacionais e Sociais, \\ Universidade Federal de \\ Pernambuco \\ cristinegusmao@gmail.com
}

\begin{abstract}
Resumo
Os fóruns de discussão online são um tipo de atividade reconhecidamente importante para a promoção do intercâmbio de ideias e de construção de conhecimento colaborativo no contexto do ensino online. A ativa participação de seus integrantes torna-se, portanto, um requisito fundamental para que a aprendizagem se dê efetivamente, pois uma diversidade de conhecimentos interagindo sinergicamente resulta em uma rica base para quem a consulta, e, mais ainda, pode ser absorvida de maneira profunda por quem participa de sua construção. Na educação por meio virtual, também há que se considerar a importância que vêm ganhando as técnicas de Inteligência Artificial (IA), que possibilitam a extração de informações relevantes em ambientes que geram uma grande quantidade de dados, dificilmente tratados por técnicas da computação convencional. Assim, este trabalho visa investigar como a literatura tem reportado a utilização da IA para fomentar a participação em fóruns online, utilizados na aprendizagem virtual. Uma revisão sistemática foi feita e, apesar de inicialmente ter sido retornada uma grande quantidade de resultados, após uma análise mais detalhada, foram selecionados apenas 18 trabalhos de fato relacionados com o objetivo de pesquisa. Conclui-se, deste fato, que a IA ainda não está sendo amplamente usada na resolução do problema da participação em fóruns educacionais, o que sugere uma área de estudo potencialmente subutilizada, que, se bem explorada, pode fornecer uma contribuição importante para auxiliar na eficácia desta ferramenta.
\end{abstract}

Palavras-Chave: Fóruns Online, Inteligência Artificial, Aprendizagem Virtual, Colaboração, Revisão Sistemática

\begin{abstract}
Online discussion forums are important to promote the exchange of ideas and the construction of collaborative knowledge in virtual learning. Thus, the active participation of its users is required to the effectiveness of the learning process, as the diversity and synergy of knowledge creates a rich base for the users and, additionally, the participants can absorb the content more deeply, in creating it. In the virtual education context, one also should consider the increasing importance of the techniques of Artificial Intelligence (AI), as they enable the extraction of relevant information in environments which use a massive number of data, not easily tackled by traditional computation techniques. This work investigates how the scientific literature reports the use of AI to promote participation in online forums used in distance learning. We conducted a systematic review, and although we obtained a great number of results in the initial search, after a more detailed analysis, only 18 articles were considered relevant to this research. The following conclusion is that AI is still being underutilized as a solution for the problem of participation in educational forums, but if it is adequately explored, it has potential to provide an important contribution to the efficacy of this tool.
\end{abstract}

Keywords: Online Forums, Artificial Intelligence, Virtual Learning, Collaboration, Systematic Review

Cite as: Mendes, P. B., Machiavelli, J. L. \& Gusmão C. M. G.de.(2019). Systematic Review of the Use of Intelligent Forums for Promoting Collaboration in Virtual Learning (Revisão Sistemática da Utilização de Fóruns Inteligentes para a Promoção da Colaboração na Aprendizagem Virtual). Brazilian Journal of Computers in Education (Revista Brasileira de Informática na Educação - RBIE), 27(3), 82-101. DOI: 10.5753/RBIE.2019.27.03.82 


\section{Introdução}

As tecnologias digitais têm permeado grande parte das atividades humanas, e com a educação não tem sido diferente. A incorporação da cultura digital ao currículo de programas educacionais é crescente (Chassignol, Khoroshavin, Klimova \& Bilyatdinova, 2018), e os recursos tecnológicos utilizados, como ambientes virtuais de aprendizagem, redes sociais, aplicativos, entre outros, estão transformando de maneira significativa as práticas e a estrutura de ensino-aprendizagem. Entre tais ferramentas, os fóruns online têm se tornado cada vez mais presentes na educação virtual (Patriarcheas \& Xenos, 2010). Eles visam proporcionar a tais ambientes um aspecto interativo e colaborativo, ao fomentar discussões, promovendo um espaço em que os participantes do processo educativo possam expor suas opiniões e ideias, em forma prioritariamente textual. A criação de um ambiente de comunicação entre vários participantes localizados remotamente, envolvendo a troca de mensagens em diversos tópicos, data do final da década de 70, com os bulletin board systems (BBS) (Ramaiah, 1995). Porém, percebe-se que mesmo após várias décadas, o formato dos fóruns, bem como suas funcionalidades e recursos agregados, pouco mudaram. No entanto, a computação vem desenvolvendo técnicas bastante poderosas, como aquelas baseadas em Inteligência Artificial (IA), que podem dotar os fóruns com a capacidade de adaptação e aprendizado através de dados gerados por seu uso e, com isso, aumentar sua eficácia, inclusive no incentivo à participação, que é um pré-requisito importante para isto.

Assim, o objetivo deste trabalho é identificar se é reportada na literatura acadêmica a utilização de IA em fóruns online na área de educação, com a finalidade de promover e ampliar a colaboração dos seus participantes.

O presente artigo está dividido da seguinte maneira: após esta introdução, serão apresentados os conceitos fundamentais, necessários para a caracterização do objeto de estudo, e a seguir será descrita a metodologia adotada. Nas seções subsequentes, serão demonstrados os resultados obtidos, a discussão e conclusão.

\section{Conceitos Fundamentais}

Para melhor compreender a proposta deste artigo, é importante esclarecer o que são ambientes virtuais de aprendizagem, as funções da colaboração e dos fóruns de discussão no ensino online e como a inteligência artificial pode ser empregada para aprimorar os processos de ensino e aprendizagem quando se pensa em colaboração em espaços virtuais.

\subsection{Ambientes Virtuais de Aprendizagem}

Nas últimas décadas, a Internet e a World Wide Web criaram um espaço alternativo, mediado por recursos tecnológicos, como computadores, tablets e smartphones, que serve para estender e substituir localidades físicas, em atividades que antes eram exclusivamente realizadas em ambientes concretos, as quais incluem compras, educação, trabalho e entretenimento (Kalay, 2004). Neste contexto, em meados da década de 90, surgiram os ambientes virtuais de aprendizagem (AVAs), com a finalidade de apoiar atividades de ensino e aprendizagem por meio da Internet (O'Leary \& Ramsden, 2002).

Os AVAs destinam-se à disponibilização e organização de informações, possibilitam o contato com mídias diversas (textos, áudios, vídeos, animações, cenários virtuais, entre outros), a realização de atividades (jogos, questionários, produções textuais, etc.), e a comunicação 
interpessoal. De acordo com Dillenbourg, Schneider e Synteta (2002), são espaços projetados de informação, mas também de interação social, nos quais a informação é representada desde a forma de texto até ambientes imersivos 3D, são cocriados pelos estudantes e podem ser utilizados não apenas para a educação a distância, mas também para enriquecer atividades presenciais, integrando múltiplas abordagens pedagógicas.

Considerando que ao utilizar os AVAs os sujeitos estarão, na maioria das vezes, separados fisicamente, as ferramentas de colaboração ganham espaço importante porque proporcionam interação entre as pessoas.

\subsection{Colaboração em e-learning}

Colaboração refere-se à troca de informações, alteração de atividades, compartilhamento de recursos e melhoria da capacidade do outro em benefício mútuo, para alcançar um objetivo comum (Himmelmann, 2002). O conceito de aprendizagem colaborativa descreve uma variedade de práticas educacionais, presentes em diversos contextos de ensino, em que a interação entre pares é o fator mais importante na aprendizagem (Dillenbourg, Järvelä \& Fischer, 2009).

Comparada a cenários em que esta é feita de forma individual ou competitiva, a aprendizagem colaborativa traz resultados mais positivos aos estudantes, aumentando suas habilidades de resolução de problemas, oferecendo vantagens cognitivas e influenciando positivamente o desenvolvimento de traços de personalidade (Hamburg, Lindecke \& Thij, 2003). Noroozi, Weinberger, Biemans, Mulder \& Chizari (2012) destacam a importância de desenvolver as capacidades de argumentação, pensamento crítico e raciocínio lógico, especialmente devido à necessidade atual de lidar com problemas complexos, e afirmam que é possível desenvolvê-las por meio de ambientes virtuais colaborativos.

Assim, na e-learning, a aprendizagem também pode ser beneficiada por meio de atividades em que há colaboração e compartilhamento entre os alunos. Eles não apenas aprendem com a participação em tais atividades, mas também por observar a forma de trabalho dos demais estudantes no ambiente web (Cheung \& Vogel, 2013). A colaboração é viabilizada por uma infraestrutura tecnológica, e sua implementação pode ser feita por meio de listas de email, fóruns online ou aplicações peer-to-peer (aprendizagem por pares) (Mason \& Lefrere, 2003). Scripts de colaboração são outra possível abordagem, a qual consiste em estruturas de apoio à interação, que especificam, sequenciam e distribuem papéis e atividades entre os participantes de um grupo (Fischer et al., 2013).

Percebe-se, portanto, que a aprendizagem colaborativa visa promover a interação entre pessoas, seja ela por meio de ferramentas síncronas ou assíncronas, de forma que os estudantes são encorajados ou solicitados a trabalhar em conjunto na construção e no compartilhamento do conhecimento (Barros, Santos \& Romero, 2019; Dantas et al. 2018).

\subsection{Fóruns Online}

Os fóruns de discussão, na educação online, são espaços de diálogo assíncrono que podem ser utilizados em ambientes virtuais de aprendizagem. Barros, Santos \& Romero (2019) referem que os fóruns proporcionam a comunicação horizontal nos AVAs. A ferramenta quando efetivamente utilizada, é capaz de aumentar o engajamento e o desenvolvimento de comunidades virtuais de aprendizagem. Por meio desta ferramenta, os envolvidos com a oferta educacional podem trocar experiências, compartilhar dúvidas e pontos de vista, fazer debates, responder a questões propostas por um facilitador ou demais participantes, receber feedback, tudo isso visando promover uma compreensão mais profunda do objeto de estudo (Siebra \& 
Machiavelli, 2015; Wise, Hausknecht \& Zhao, 2014). As informações são armazenadas em um espaço virtual, o que permite que elas possam ser recuperadas e revisadas a qualquer momento (Balaji \& Chakrabarti, 2010).

Kanuka (2005) afirma que este tipo de atividade permite a realização de uma aprendizagem em um nível mais elevado, favorecendo o pensamento crítico, além de melhorar a qualidade da experiência de aprendizado como um todo, o que é corroborado por Kolb (1984), que associa a participação regular em discussões ao aprimoramento do processo de aprendizagem. Isto pode ser comprovado pela correlação entre o aumento das notas dos estudantes com sua participação em fóruns de discussão, conforme observado por Webb, Jones, Barker \& van Schaik (2004).

No entanto, para que os fóruns ofereçam os benefícios descritos, é preciso que eles tenham um bom nível de participação. Mesmo em fóruns que possuem participação obrigatória, é comum o problema de os usuários interagirem apenas para cumprir os requisitos mínimos e abandonar a discussão, sem acompanhar e participar das demais postagens, o que pode reduzir a eficácia da ferramenta. Portanto, estratégias que possibilitem um melhor monitoramento da participação nos fóruns, bem como estimulem a interação entre os participantes, possíveis de ser implementadas com uso de técnicas de Inteligência Artificial, são capazes de potencializar o uso desta ferramenta.

\subsection{Inteligência Artificial (IA)}

A inteligência artificial é uma área de estudo, definida por McCarthy et al. (2006), baseada na conjectura que todo aspecto do aprendizado ou qualquer outra característica de inteligência podem ser tão precisamente descritos que uma máquina pode ser construída de forma a simulálos, como por exemplo, nas capacidades de usar linguagem, formar abstrações e conceitos, resolver problemas reservados para humanos e aprimorar a si mesma. Conforme Russell e Norvig (1995), a engenharia da computação, com seus avanços, incluindo a velocidade de processamento e memória, viabilizou a implementação destas funcionalidades em máquinas digitais.

A IA compreende um conjunto de técnicas que podem ser aplicadas em uma ampla gama de situações, como por exemplo, o processamento de linguagem natural, busca inteligente em bases de dados, sistemas especialistas, prova de teoremas, robótica, programação automática, problemas combinatoriais e problemas de percepção do ambiente (Nilsson, 2014). Alguns exemplos apresentados por Russell e Norvig (1995) incluem sistemas de reconhecimento de voz, sistemas de monitoramento em tempo real, mecanismos de direção automatizada e sistemas de auxílio ao diagnóstico de doenças. Tais casos demonstram como atividades, a princípio, de atribuição exclusiva aos humanos, com o advento da IA, têm tido sua execução possibilitada por sistemas computacionais, o que permite que se trate a grande quantidade de informações envolvidas em cada uma delas, e de forma adaptável.

No âmbito da educação, a incorporação de técnicas de IA data do início da década de 70 (Nwana, 1990), e o objetivo geral de sua aplicação é a personalização e suporte da aprendizagem, auxiliando na melhoria da experiência do estudante (Zaidi, Shane \& Hannah, 2019).

Atualmente, tem sido utilizada em diversos níveis, desde o ensino fundamental até as universidades, incluindo ferramentas e tecnologias como robôs, sistemas tutores inteligentes e sistemas adaptativos de aprendizagem (Chassignol et al., 2018). Zaidi et al. (2019) mencionam, além destes recursos, a aplicação de IA nos seguintes cenários: aprendizagem baseada em jogos, 
onde também podem ser utilizados simuladores, para uma experiência mais engajadora; ambientes de aprendizagem interativos, auxiliando a mediar discussões online, inclusive com o uso de bots; e modelos de dados de estudantes, identificando correlações entre as ações do usuário e seu desempenho. Em fóruns online, a IA tem sido usada para a análise dos discursos presentes nas mensagens trocadas entre os participantes (Conde, 2008), mas o presente artigo também identifica a possibilidade de fomento à participação, como é exemplificado na seção de Resultados.

\section{Metodologia}

Segundo Kitchenham (2004), uma revisão sistemática é um meio de identificar, avaliar e interpretar toda a pesquisa disponível sobre uma determinada questão de pesquisa, tópico ou fenômeno. Esta metodologia sintetiza os trabalhos gerados em uma determinada área de estudo, selecionados a partir de critérios que sejam relevantes para a abordagem em questão. A busca de tais trabalhos, em geral, é realizada em bases de dados acadêmicas, que são compreendidas como fontes de informação eletrônicas, pesquisáveis de modo interativo ou conversacional através de um computador (Silva, Ramos \& Noronha, 2006). A utilização de meio eletrônico para a pesquisa possibilita a localização de uma grande quantidade de trabalhos, publicados em todo mundo, o que é necessário para um bom embasamento do estudo, além de propiciar uma maior agilidade e conveniência, com relação à pesquisa de itens disponíveis em meio físico. Neste artigo, foram selecionadas as seguintes fontes para a pesquisa de trabalhos relacionados ao tema:

- Scopus

- ProQuest

- ScienceDirect

- IEEE Xplore

- ACM Digital Library

- Springer Link

Elas foram escolhidas por permitirem encontrar diversas publicações na área de educação e tecnologia, tendo potencial para fornecer uma boa amostra para este estudo. Optou-se em restringir as publicações a serem avaliadas pelas que usam o idioma inglês, que é o principal utilizado na literatura científica internacional (Van Weijen, 2012).

\subsection{Questões de Pesquisa (QP)}

O trabalho aqui apresentado visou responder às seguintes questões:

- QP1: É possível encontrar, na literatura acadêmica, registros da utilização de fóruns online que utilizem a Inteligência Artificial para promover uma maior colaboração de seus usuários, no contexto da educação a distância?

- QP2: Quais as principais técnicas de Inteligência Artificial aplicadas em fóruns usados com finalidade educativa?

\subsection{String de Busca}


As bases de dados extraem itens a partir do fornecimento de uma string de busca, isto é, uma cadeia de caracteres formada por um ou mais termos, relacionados por operadores lógicos. Estes indicam de que forma os termos devem ser recuperados, o que é detalhado a seguir:

- Operador AND: Indica que o item recuperado deve conter todos os termos ligados por ele.

- Operador OR: Indica que o item recuperado deve conter pelo menos um dos termos ligados por ele.

- Operador NOT: Indica que o item recuperado não poderá conter o termo precedido dele.

Apesar de algumas bases de dados possuírem recursos específicos para refinar as buscas, optou-se por utilizar apenas estes operadores simples, que são válidos em todas elas.

Além dos operadores, é fundamental que se defina adequadamente os termos a compor a string, de forma a recuperar os trabalhos que mais se aproximam semanticamente ao objeto de estudo. A Tabela 1 mostra os termos selecionados para a string de busca utilizada neste trabalho, mostrada abaixo:

(forum OR asynchronous) AND (collaborative OR collaboration OR CSCL) AND ("artificial intelligence" OR intelligent OR "machine learning") AND (e-learning OR "distance learning" OR "online learning").

Tabela 1: Termos da string de busca.

\begin{tabular}{|l|l|}
\hline Termo & Propósito \\
\hline forum OR asynchronous & $\begin{array}{l}\text { Identificar trabalhos relacionados com fóruns. Como o fórum é o } \\
\text { tipo de atividade assíncrona mais comum em ambientes online, } \\
\text { este termo também foi adicionado como um sinônimo. }\end{array}$ \\
\hline $\begin{array}{l}\text { collaborative OR collaboration OR CSCL } \\
\text { "machine learning" }\end{array}$ & Identificar o aspecto colaborativo. \\
\hline $\begin{array}{l}\text { e-learning OR "distance learning" OR "online } \\
\text { learning" }\end{array}$ & $\begin{array}{l}\text { Identificar a relação com a Inteligência Artificial. Machine } \\
\text { learning é uma sub-área desta disciplina, de grande relevância, } \\
\text { então este termo também foi incluído. }\end{array}$ \\
\hline
\end{tabular}

\subsection{Critérios de Seleção de Trabalhos Científicos}

Com a busca supracitada, foram recuperados 5176 trabalhos. Na Tabela 2, é mostrada a quantidade de publicações encontradas em cada fonte de dados. A maioria dos trabalhos foram localizados no Scopus, o que se justifica por se tratar de um motor de busca que faz pesquisa em diversas bases de dados científicas.

Tabela 2: Quantidade de trabalhos encontrados por fonte de dados.

\begin{tabular}{|c|c|c|}
\hline Fonte & Quantidade & Percentual do total \\
\hline ProQuest & 1217 & $23,51 \%$ \\
\hline Science Direct & 216 & $4,17 \%$ \\
\hline Scopus & 3298 & $63,72 \%$ \\
\hline ACM Digital Library & 37 & $0,71 \%$ \\
\hline Springer Link & 407 & $7,86 \%$ \\
\hline
\end{tabular}


Foi preciso definir critérios para reduzir a quantidade de trabalhos a serem analisados e identificar quais de fato tinham relevância para a presente pesquisa. A seguir, são demonstrados os critérios adotados:

- Critérios de inclusão: Artigos completos, publicados em periódicos, revistas e capítulos de livro, em inglês, relacionados com tecnologia educacional. Segundo Cronin, Ryan \& Coughlan (2008), o período a ser adotado em revisões de literatura, em geral, deve compreender os últimos 5 a 10 anos de pesquisa. Como o presente trabalho está enquadrado na área de tecnologia, em que são frequentes as mudanças no estado da arte, optou-se por incluir artigos a partir de 2014.

- Critérios de exclusão: Artigos que não atendam aos critérios de inclusão, duplicados e cujo acesso não possa ser realizado de forma gratuita ou pelo portal de periódicos da CAPES.

A partir destes critérios, obteve-se 387 trabalhos, dos quais se analisou os títulos e resumos, reduzindo a quantidade de artigos para leitura completa a 109 itens.

\subsection{Coleta e Análise de Dados}

Para a análise dos artigos selecionados, foram extraídas as seguintes informações, consideradas relevantes para a resolução das perguntas de pesquisa: nome do artigo, autores, país em que o estudo foi realizado, ano da publicação, palavras-chave, ambiente de aplicação, nível de ensino e técnica de IA abordada. Além disso, foram observados dados a respeito das publicações, a seguir: nome, área e fonte da qual foi recuperada.

\section{Resultados e Discussão}

O processo de refinamento dos resultados obtidos por meio da metodologia aplicada está sumarizado na Figura 1. Após a realização da etapa de leitura integral dos artigos préselecionados, resultaram 18 artigos, com os quais se procedeu a coleta e organização de informações mais detalhadas, sintetizadas na tabela disponível no Apêndice. 


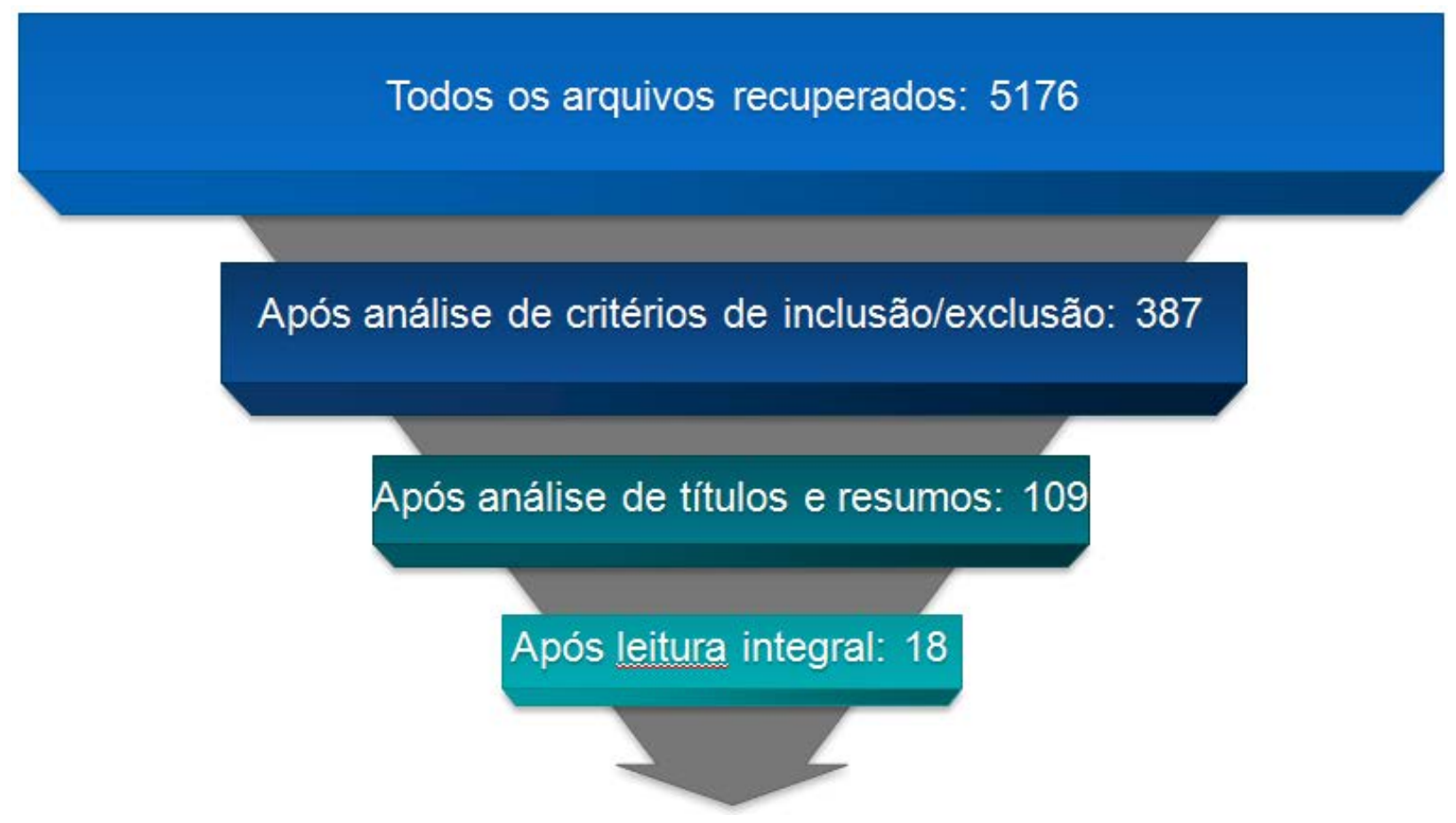

Figura 1: Refinamento dos resultados de busca.

\section{1 Origem e Data dos Estudos}

Observou-se uma boa distribuição dos estudos quanto a seus países de origem, contemplando quase todos os continentes, com exceção da Oceania. Isto mostra que a temática abordada nesta revisão vem sendo estudada tanto em contextos em que o nível de desenvolvimento é maior, quanto em países ainda por se desenvolver economicamente. A Espanha é o país que apresenta mais artigos, 5 no total, e destaca-se a presença de três estudos da Open University of Catalonia, a primeira universidade virtual da Espanha, o que demonstra a importância da ferramenta de fóruns online para esta instituição, visto que ela oferece apenas educação a distância (Caballé et al., 2014). Outra observação a ser feita é a ausência de estudos brasileiros nos trabalhos recuperados, o que indica que os pesquisadores brasileiros de educação a distância ainda não estão dialogando com esta temática no cenário internacional. A Figura 2 ilustra a distribuição de artigos por continente.

Sobre a data em que eles foram realizados, percebe-se uma maior concentração de artigos publicados no ano de 2014. Esta pesquisa foi realizada até agosto de 2019, portanto, no que se refere a este ano, poderá haver omissão de trabalhos publicados em momento posterior a realização desta revisão. O gráfico apresentado na Figura 3 mostra a relação de quantidade de publicações por ano, e apresenta uma tendência decrescente em relação ao início do período considerado neste artigo. Uma possível explicação para esse fato é que, segundo Malik (2017), as áreas de IA aplicada à educação, notadamente Learning Analytics e mineração de dados educacionais (Educational Data Mining), têm priorizado o suporte e aprimoramento da inteligência humana, o que pode ter tirado a atenção da investigação de mecanismos automáticos para a melhoria da experiência de aprendizagem, como são os casos tratados neste artigo. 


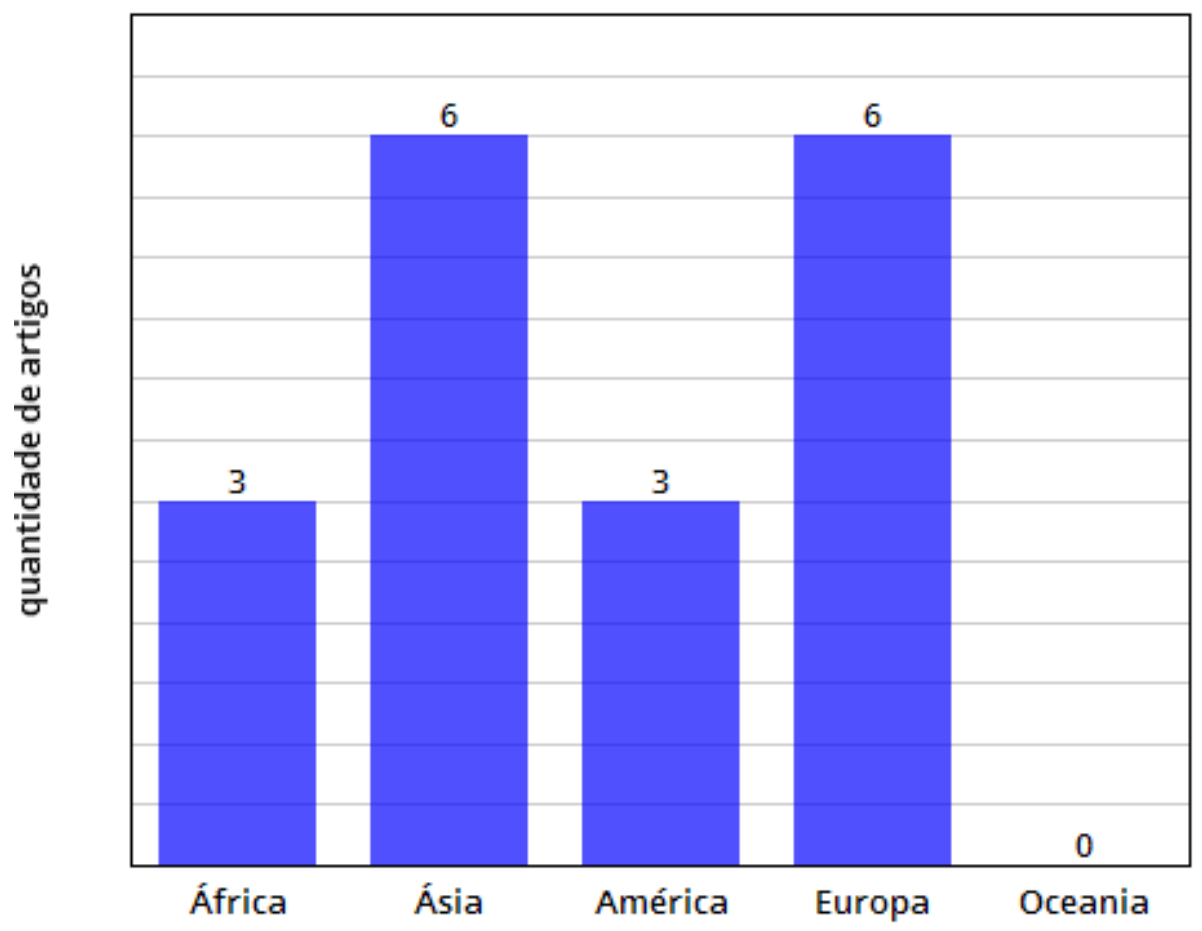

Figura 2: Distribuição de artigos por continente.

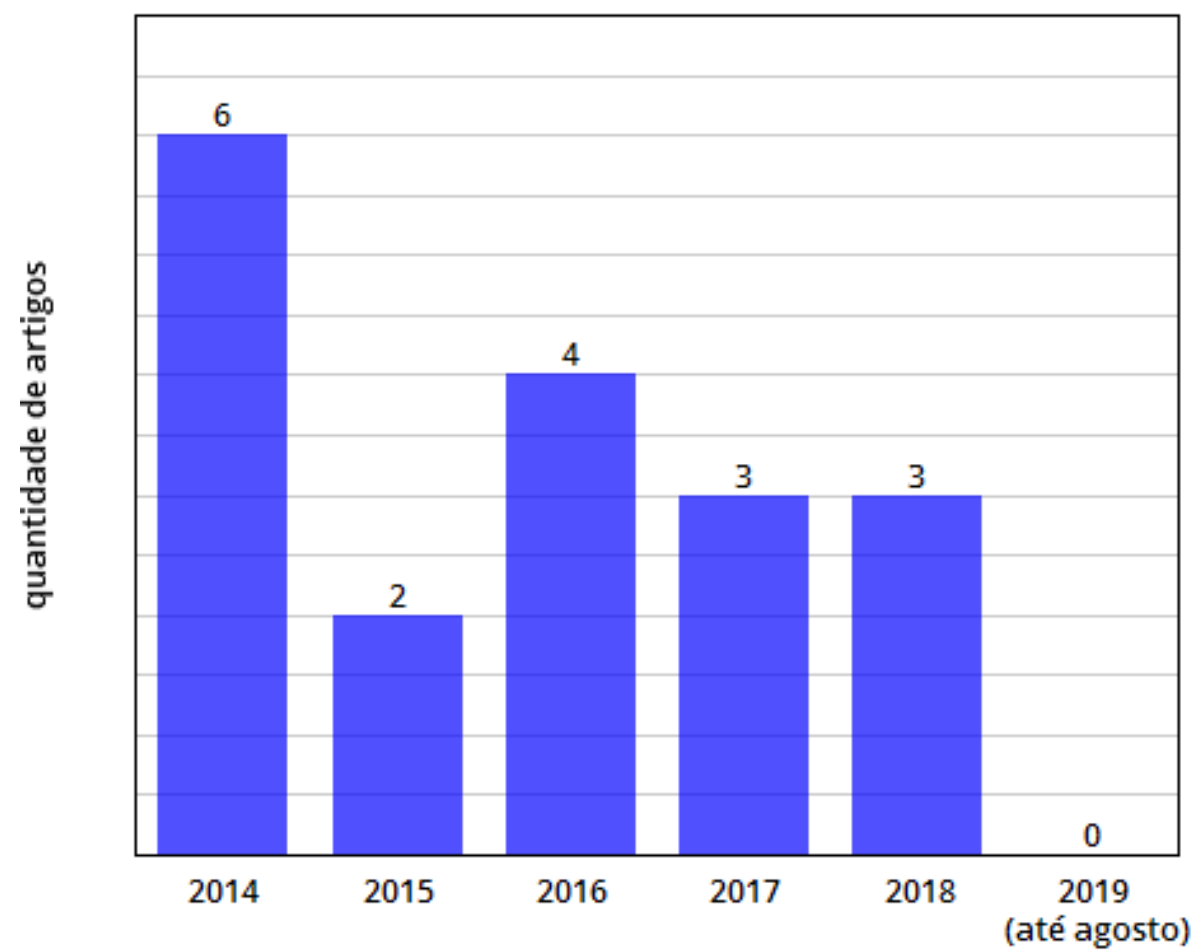

Figura 3: Distribuição de artigos por ano.

\section{2 Contextos de Aplicação}

Foi verificado que a maioria dos estudos foi aplicada no ensino de nível superior, apresentando como exceções um caso de ensino de idiomas (Troussas, Virvou \& Alepis, 2014), outro em que 
é utilizado um web fórum de propósito geral (Albatayneh, Ghauth \& Chua, 2014), e ensino médio (Arguedas, Xhafa, Casillas, Daradoumis \& Peña, 2016). Isto é consistente com a constatação de Kadagidze (2014), que relata que os fóruns online têm sido amplamente utilizados no ensino superior para promover a educação a distância ou complementar discussões presenciais. Sobre o meio tecnológico utilizado, os mais frequentes foram os AVAs baseados na plataforma Moodle, um sistema open-source adotado em dezenas de milhares de ambientes de aprendizagem em todo o mundo, sendo o mais amplamente utilizado (Moodle, 2018).

Outras plataformas de AVA contempladas foram o Intelligent Web Teacher (IWT) e dotLRN. Também foram encontrados trabalhos que utilizaram um fórum online baseado em phpBB, que é líder em utilização na categoria de sistemas de bulletin board de código aberto, sendo uma solução de ampla flexibilidade de uso (phpBB Limited, 2014), o que explica sua popularidade.

\subsection{Técnicas de Inteligência Artificial}

A técnica de IA mais frequentemente utilizada nos artigos selecionados foi o processamento de linguagem natural, definido por Liddy (2001) como um conjunto de técnicas computacionais que analisam e representam textos que ocorrem naturalmente, em um nível de análise linguística com o propósito de atingir um nível de processamento comparável ao de humanos, para uma gama de tarefas ou aplicações. Os fóruns online são ferramentas que prioritariamente se baseiam em textos escritos, os quais geralmente não possuem nenhuma estrutura, sendo de livre criação pelos usuários, assim, as técnicas de processamento de linguagem natural possuem uma maior flexibilidade para lidar com as variações da linguagem humana, o que justifica sua adoção em diversos trabalhos deste estudo. Mais especificamente, chama a atenção a utilização da técnica de Análise de Semântica Latente, que é um modelo utilizado para analisar um corpo de texto natural, gerando uma representação que captura a similaridade de palavras e passagens de texto (Landauer \& Dumais, 1997). A capacidade de processar uma grande quantidade de informação é um requisito importante nos fóruns online, o que é contemplado por esta técnica.

Outra vertente de técnicas encontrada foram métodos de classificação e clusterização, que objetivam o aprendizado de características de um conjunto de dados, separando-os em grupos (Linden, 2009).

Algo notável é que apenas um trabalho dentre os selecionados, de autoria de Njenga, Oboko, Omwenga \& Maina (2017), faz uso de agentes inteligentes. Estes são definidos como softwares autônomos que se comunicam através do envio e recebimento de mensagens num determinado ambiente (Njenga et al., 2017). Por serem flexíveis e interativos, são compatíveis com a natureza dos fóruns, que envolvem uma vasta e diversa troca de informações, constituindo-se em um contexto bastante dinâmico.

\subsection{Estratégias de Promoção da Colaboração}

Ao se analisar como os estudos incluídos nesta revisão sistemática trataram a questão de como promover uma maior colaboração em fóruns online, foram identificados cinco tipos de estratégias, descritos a seguir:

- Apresentação diferenciada do conteúdo do fórum - Nestes casos, a apresentação do conteúdo de forma textual foi modificada para se tornar mais atrativa ou mais simples de ser processada cognitivamente. Em Caballé et al. (2014), Caballé et al. (2014a) e Mora et al. (2016), foi desenvolvido um recurso de visualização de sessões colaborativas, utilizando avatares como meio de interação com o usuário. Esta interação é baseada na técnica de análise de sentimentos, que permite que sejam extraídas as emoções 
associadas aos textos postados no fórum, o que ocasiona uma melhoria da experiência do usuário, na medida em que a resposta do avatar reflete a emoção que aquele expressou, gerando empatia. Arguedas et al. (2016) criaram uma estratégia bastante semelhante a estes outros trabalhos, também utilizando avatares para prover uma resposta ao usuário, mas desta vez, baseando-se em lógica Fuzzy. Este tipo de classificação provê flexibilidade na captura de emoções dos usuários, relacionando-as com um dicionário afetivo, e permite uma resposta apropriada em cada caso. Já em Al-Mousa e Fiaidhi (2014), criou-se uma funcionalidade de síntese de conteúdo das discussões, a qual utiliza processamento de linguagem natural e algoritmos de poda. Estas técnicas permitem a extração de informações relevantes de conteúdos textuais, o que facilita o compartilhamento das ideias, na medida em que diminui a sobrecarga cognitiva do usuário, já que ele terá menos informação para processar.

- Elementos de dinamização - Elementos externos às funcionalidades do fórum podem ser adicionados para auxiliar em sua dinâmica. Njenga et al. (2017) descreveu o uso de agentes inteligentes para facilitar a colaboração em fóruns na plataforma Moodle. No trabalho de Troussas et al. (2014), além de o próprio meio de utilização, via celular, intencionar a facilitação das interações, foram incorporados elementos de avaliação e classificação de perfis de alunos, inseridos para dinamizar a resolução de questões de forma colaborativa.

- Auxílio ao diagnóstico de níveis de colaboração - Permitem a identificação do nível de colaboração dos usuários no fórum, indicando pontos de melhoria. Anaya et al. (2016) propõem uma ferramenta de análise visual, que busca provocar a autorreflexão e embasa uma recomendação de ação de acordo com o status identificado para o usuário. A proposta de Saqr et al. (2018) é utilizar Análise de Redes Sociais para inferir o grau de interação dos usuários. Essa informação é extraída através da detecção de clusters, formados com base nas ações que os usuários realizam no fórum, o que permite um diagnóstico que indica se o nível de participação está adequado, e informa uma possível intervenção, a depender da necessidade. Abordagem similar é encontrada no sistema de aprendizagem colaborativa descrito no trabalho de Ji, Park, Jo \& Lim (2015), que além das redes de relacionamento dos usuários, analisa seus níveis de engajamento e aprendizagem, disponibilizando estes dados para tutores, estudantes e administradores. É válido destacar que estes casos se constituem em uma estratégia indireta, visto que ela não promove uma maior interação por si só, mas ela possibilita que o instrutor tome conhecimento de um nível de colaboração aquém do esperado e assim possa agir de acordo.

- Formação de grupos - Neste tipo de estratégia, os usuários são alocados em grupos, designados para que seus integrantes sejam mais compatíveis e/ou complementares, facilitando as interações. Um mecanismo de formação automática de grupos em um sistema tutor inteligente, baseado no algoritmo de busca local 2-opt, foi abordado por Salihoun, Guerouate, Berbiche \& Sbihi (2017), obtendo sucesso no incremento da participação no fórum. Maina et al. (2017) desenvolveu uma solução de agrupamento com base no grau de colaboração, utilizando aprendizado de máquina e algoritmos kmédias e Expectation-maximization. Foram obtidos resultados positivos tanto nas interações sociais quanto no nível de aprendizagem.

- Recomendação de postagens e/ou usuários - São mecanismos que identificam as preferências dos usuários e fazem recomendações congruentes com estas. Para avaliar quais itens a serem recomendados são mais adequados às características dos usuários em questão, esta estratégia pode fazer uso de técnicas de classificação e clusterização. Um exemplo é o sistema de auxílio entre pares iHelp, descrito por Vassileva, McCalla \& 
Greer (2016), que faz uso da clusterização para identificar as relações dos estudantes com os conceitos do curso, e assim indicar os usuários mais adequados para auxiliá-lo em suas questões no fórum (Greer et al., 1998). Albatayneh et al. (2014) propõe uma arquitetura de sistema de recomendação baseada na análise da semântica de conteúdo do fórum, técnica também utilizada por Kim e Shaw (2014), em uma ferramenta de recomendação de discussões passadas. Esta abordagem facilita a interação na medida em que traz à tona tópicos que poderiam estar em desuso, o que contribui para a continuidade da discussão. Numa proposta mais diferenciada, Albatayneh et al. (2018) realiza a recomendação de postagens baseando-se não apenas em seu conteúdo, mas também nas avaliações negativas fornecidas pelos usuários, o que aumentou a qualidade da recomendação e os resultados dos estudantes. A recomendação de usuários está presente no trabalho de Al-Dhanhani, Mizouni, Otrok \& Al-Rubaie (2015), onde eles descrevem um sistema social de aprendizagem customizado que usa os dados do perfil de usuário para realizar as sugestões, que permitem que o estudante encontre potenciais colaboradores. Chao, Lai, Liu \& Lin (2018), por sua vez, apresentam uma proposta de fórum que inclui a recomendação de usuários especialistas, com o objetivo de responder às questões colocadas pelos participantes e, assim, aumentar sua motivação e participação no processo de aprendizagem.

\section{5 Limitações da Pesquisa}

Durante o processo de escrita deste trabalho, algumas limitações foram encontradas. A principal foi que a seleção dos artigos foi executada por apenas um pesquisador, o qual pode possuir vieses que o levaram a excluir artigos potencialmente relevantes para a pesquisa. Além disso, a decisão de utilizar apenas operadores lógicos simples na busca nas bases de dados pode ter tornado menos eficiente a recuperação de publicações relevantes. Por fim, os próprios termos utilizados na pesquisa são facilmente encontrados em contextos não relacionados com o tema específico deste artigo, o que fez com que a quantidade de resultados iniciais fosse excessivamente alta, e que fosse necessário rejeitar a maior parte deles seguindo outros critérios, como a leitura do título ou verificação da área da publicação, por exemplo.

\section{Conclusões}

O presente trabalho objetivou identificar estudos na literatura acadêmica que abordem a questão do incentivo à colaboração em fóruns online por meio de métodos de Inteligência Artificial para solucioná-la. Foi realizada uma revisão sistemática da literatura, compreendendo artigos escritos na língua inglesa, no período de 2014 a agosto de 2019. As fontes de dados consultadas para a obtenção destes foram: ProQuest, Science Direct, Scopus, IEEE Xplore, ACM Digital Library e Springer Link. Elas foram selecionadas por possuírem uma boa reputação e terem diversas publicações nas áreas de Educação e Tecnologia, em cuja interseção se encontra o tema deste artigo. A busca inicial, considerando termos relacionados com fórum, colaboração e Inteligência Artificial, forneceu 5176 resultados, que após a aplicação de critérios de inclusão e exclusão e análise de títulos e resumos, foram reduzidos para 109 artigos dos quais se procedeu uma leitura completa. Por fim, 18 destes trabalhos foram incluídos nesta revisão sistemática.

Foram identificados trabalhos de origens geográficas diversificadas, contemplando quase todos os continentes. Também foi observada uma predominância de publicações do ano de 2014, o que indica um decréscimo da exploração deste tema, dentro do recorte observado, o que pode ser explicado pelo foco da pesquisa de IA aplicada à educação estar sendo mais voltado ultimamente para suporte aos elementos humanos envolvidos, e não tanto à melhoria de 
ferramentas de aprendizagem, como os fóruns. Em outras palavras, na educação, seguindo a tendência de outras áreas, a IA tem se concentrado em estudos que envolvem big data, com extração de dados em ambientes virtuais de aprendizagem. Isso indica que parece haver uma prioridade a ferramentas de diagnóstico, mais direcionadas aos gestores de cursos, ao invés das ferramentas que de fato são utilizadas como importantes recursos pedagógicos e que pouco evoluíram ao longo dos anos, apesar dos recursos tecnológicos disponíveis. Para que haja uma maior expansão no estudo da incorporação das tecnologias nas atividades didáticas, como é o caso dos fóruns, sugere-se um maior envolvimento dos pedagogos e designers instrucionais, em colaboração com as equipes de desenvolvimento de software. Assim, poderão trabalhar de forma sinérgica, adequando os potenciais das técnicas computacionais às necessidades pedagógicas sinalizadas pelos profissionais da educação.

O contexto de aplicação mais frequente foi no ensino superior, em ambientes virtuais de aprendizagem estabelecidos em sistemas específicos para esse fim, como a plataforma Moodle. Sobre as técnicas de Inteligência Artificial apresentadas, observou-se uma maior utilização de métodos envolvendo Processamento de Linguagem Natural, um resultado esperado pela característica dos fóruns de lidar predominantemente com informação em forma de texto, produzido por humanos. Também destacaram-se, com menor frequência, métodos de classificação e clusterização, associados a funcionalidades de diagnóstico e recomendação. Diversas estratégias de incentivo à colaboração foram implementadas por meio destas técnicas, as quais foram classificadas de maneira mais genérica em: apresentação diferenciada do conteúdo do fórum, elementos de dinamização, diagnóstico do grau de colaboração, formação de grupos de estudantes e recomendação de postagens.

Conclui-se, respondendo à primeira pergunta de pesquisa, que foram encontrados trabalhos científicos que utilizam a IA na promoção da colaboração, no entanto, a sua quantidade ainda é escassa, ao se estimar o potencial que esta tecnologia pode oferecer. Esperase que o presente artigo seja uma contribuição para indicar possíveis aplicações deste tipo de recurso computacional em fóruns online, e a perspectiva futura é que esta revisão sistemática embase o desenvolvimento de uma ferramenta de fórum que faça uso da IA para ampliar o nível de engajamento e colaboração de seus participantes de duas formas: i) subsidiando os professores e mediadores com informações que possibilitem conhecer, facilmente, as necessidades dos participantes, como os temas mais discutidos, usuários que se destacam nas discussões e a localização geográfica dos participantes; ii) estimulem a colaboração entre as pessoas ao recomendar usuários com interesses afins e até mesmo materiais que possam contribuir com os debates em andamento ou promover novas discussões.

\section{Agradecimentos}

Este trabalho foi desenvolvido a partir de pesquisa financiada pelo CNPq (Conselho Nacional de Desenvolvimento Científico e Tecnológico), ao qual se agradece o apoio prestado.

\section{Referências}

Albatayneh, N., Ghauth, K. \& Chua, F. (2014). A Semantic Content-Based Forum Recommender System Architecture Based on Content-Based Filtering and Latent Semantic Analysis. In Herawan T., Ghazali R., \& Deris M. (Eds) Recent Advances on Soft 
Computing and Data Mining. Advances in Intelligent Systems and Computing (Vol. 287). Springer, Cham. DOI: https://doi.org/10.1007/978-3-319-07692-8_35 [GS Search]

Albatayneh, N., Ghauth, K. \& Chua, F. (2018). Utilizing Learners' Negative Ratings in Semantic Content-based Recommender System for e-Learning Forum. Journal of Educational Technology \& Society, 21(1), 112-125. [GS Search]

Al-Dhanhani, A., Mizouni, R., Otrok, H. \& Al-Rubaie, A. (2015). Analysis of collaborative learning in social network sites used in education. Social Network Analysis and Mining (5)1, 1. DOI: https://doi.org/10.1007/s13278-015-0303-z [GS Search]

Al-Mousa, M. \& Fiaidhi, J. (2014). Developing a Collaborative MOOC Learning Environment utilizing Video Sharing with Discussion Summarization as Added-Value. International Journal of Multimedia and Ubiquitous Engineering, 9(11), 397-408. DOI: https://doi.org/10.14257/ijmue.2014.9.11.38 [GS Search]

Anaya, A., Luque, M., \& Peinado, M. (2016). A visual recommender tool in a collaborative learning experience. Expert Systems with Applications, 45, 248-259. DOI: https://doi.org/10.1016/j.eswa.2015.01.071 [GS Search]

Arguedas, M., Xhafa, F., Casillas, L., Daradoumis, T., Peña, A. \& Caballé, S. (2016). A model for providing emotion awareness and feedback using fuzzy logic in online learning. Soft Computing, (22)3, 963-977. DOI: https://doi.org/10.1007/s00500-016-2399-0 [GS Search]

Balaji, M. \& Chakrabarti, D. (2010). Student Interactions in Online Discussion Forum: Empirical Research from 'Media Richness Theory' Perspective. Journal of Interactive Online Learning, 9(1). [GS Search]

Barros, D., Santos, V. \& Romero, C. (2019). Estratégias para o trabalho colaborativo: revisitando o uso de fóruns on-line na educação a distância. Revista Diálogo Educacional, 19(60). DOI: http://dx.doi.org/10.7213/1981-416X.19.060.DS10 [GS Search]

Caballé, S., Gañán, D., Gómez, M., Conesa, J., Rius, A. \& Santanach, F. (2014). Experiences at the Open University of Catalonia with the Virtualization of Live Collaborative Learning in Support for Engineering Education. International Journal of Engineering Education, 30(3), 663-674. [GS Search]

Caballé, S., Mora, N., Feidakis, M., Gañán, D., Conesa, J., Daradoumis, T. \& Prieto, J. (2014a). CC-LR: providing interactive, challenging and attractive collaborative complex learning resources. Journal of Computer Assisted Learning, 30(1), 51-67. DOI: https://doi.org/10.1111/jcal.12021 [GS Search]

Chao, P., Lai, K., Liu, C., \& Lin, H. (2018). Strengthening Social Networks in Online Discussion Forums to Facilitate Help Seeking for Solving Problems. Journal of Educational Technology \& Society, 21(4), 39-50. [GS Search]

Chassignol, M., Khoroshavin, A., Klimova, A. \& Bilyatdinova, A. (2018). Artificial Intelligence trends in education: a narrative overview. Procedia Computer Science, 136, 16-24. DOI: https://doi.org/10.1016/j.procs.2018.08.233 [GS Search]

Cheung, R. \& Vogel, D. (2013). Predicting user acceptance of collaborative technologies: An extension of the technology acceptance model for e-learning. Computers \& Education, 63, 160-175. DOI: https://doi.org/10.1016/j.compedu.2012.12.003 [GS Search] 
Conde, D. (2008). A alternância da referência do sujeito-enunciador como expressão da identidade e seus efeitos de sentido. 2008 (Doctoral dissertation, Tese (Doutorado em Estudos da Linguagem) - Universidade Estadual de Londrina, Londrina). [GS Search]

Cronin, P., Ryan, F. \& Coughlan, M. (2008). Undertaking a literature review: a step-by-step $\begin{array}{llll}\text { approach. } & \text { British Journal of Nursing, 17(1), 38-43. DOI: }\end{array}$ https://doi.org/10.12968/bjon.2008.17.1.28059 [GS Search]

Dantas, A., Viana, H., Abijaude, J. \& Sobreira, P. (2018). Internet das Coisas e Aprendizagem Colaborativa: Revisão Sistemática da Literatura. In Brazilian Symposium on Computers in Education (Simpósio Brasileiro de Informática na Educação-SBIE) , 29(1), p. 278. DOI: https://doi.org/10.5753/cbie.sbie.2018.278 [GS Search]

Dillenbourg, P., Järvelä, S. \& Fischer, F. (2009). The evolution of research on computersupported collaborative learning. In Technology-enhanced learning (pp. 3-19). Springer, Dordrecht. DOI: https://doi.org/10.1007/978-1-4020-9827-7_1 [GS Search]

Dillenbourg, P., Schneider, D. \& Synteta, P. (2002). Virtual learning environments. In 3rd Hellenic Conference Information \& Communication Technologies in Education (pp. 3-18). Greece: Kastaniotis Editions. [GS Search]

Fischer, F., Kollar, I., Stegmann, K., Wecker, C., Zottmann, J. \& Weinberger, A. (2013). Collaboration scripts in computer-supported collaborative learning. The international handbook of collaborative learning, 403-419. DOI: https://doi.org/10.4324/9780203837290.ch23 [GS Search]

Hamburg, I., Lindecke, C. \& Thij, H. (2003). Social aspects of e-learning and blending learning methods. Proceedings of the fourth European conference on E-commerce, E-work, Elearning, E-health, E-banking, E-business, on-line services, virtual institutes, and their influences on the economic and social environment (E-Comm-Line), pp. 11-15. [GS Search]

Himmelmann, A. (2002). Collaboration for a change: Definitions, models, roles and a guide for collaborative process. Minneapolis: Hubert Humphrey Institute of Public Affairs, University of Minnesota. [GS Search]

Greer, J., McCalla, G., Cooke, J., Collins. J, Kumar, V. Bishop, A. Vassileva, J. (1998). The Intelligent Helpdesk: Supporting Peer-Help in a University Course. ITS 1998: Intelligent Tutoring Systems, pp. 494-503. DOI: https://doi.org/10.1007/3-540-68716-5_55 [GS Search]

Ji, H., Park, K., Jo, J. \& Lim, H. (2015). Mining students activities from a computer supported collaborative learning system based on peer to peer network. Peer-to-Peer Networking and Applications, (9)3, 465. DOI: https://doi.org/10.1007/s12083-015-0397-0 [GS Search]

Kadagidze, L. (2014). The role of forums in online instruction. European Scientific Journal, ESJ, 10(10). [GS Search]

Kalay, Y. (2004). Virtual learning environments. Journal of Information Technology in Construction (ITcon), 9(13), 195-207. [GS Search]

Kanuka, H. (2005). An exploration into facilitating higher levels of learning in a text-based Internet learning environment using diverse instructional strategies. Journal of Computer Mediated Communication, 10(3). DOI: https://doi.org/10.1111/j.1083-6101.2005.tb00256.x [GS Search] 
Kitchenham, B. (2004). Procedures for undertaking systematic reviews. Technical Report TR/SE-0401, Department of Computer Science, Keele University and National ICT, Australia Ltd. Joint Technical Report. [GS Search]

Kim, J. \& Shaw, E. (2014). Scaffolding student online discussions using past discussions: PedaBot studies. Artificial Intelligence Review, 41(1), 97-112. DOI: https://doi.org/10.1007/s10462-011-9300-4 [GS Search]

Kolb, D. (1984). Experiential Learning: Experience as the Source of Learning and Development. Englewood Cliffs, NJ: Prentice-Hall. DOI: https://doi.org/10.1002/job.4030080408 [GS Search]

Landauer, T. \& Dumais, S. (1997). A solution to Plato's problem: The latent semantic analysis theory of acquisition, induction, and representation of knowledge. Psychological review, 104(2), 211. DOI: https://doi.org/10.1037//0033-295x.104.2.211 [GS Search]

Liddy, E. (2001). Natural language processing. Encyclopedia of Library and Information Science (2nd ed.). NY: Marcel Decker, Inc. [GS Search]

Linden, R. (2009). Técnicas de Agrupamento. Revista de Sistemas de Informação da FSMA,4, 18-36. [GS Search]

Maina, E., Oboko, R. \& Waiganjo, P. (2017). Using Machine Learning Techniques to Support Group Formation in an Online Collaborative Learning Environment. International Journal of Intelligent Systems \& Applications, 9(3). DOI: https://doi.org/10.5815/ijisa.2017.03.04 [GS Search]

Malik, M. (2017). Meta-analysis and review of the use of Artificial Intelligence and Learning Analytics within Engineering Education at University level. In 5th EERN Symposium: Time for a change! Challenging the status quo in engineering education, London. [GS Search]

Mason, J. \& Lefrere, P. (2003). Trust, collaboration, e-learning and organisational transformation. International Journal of Training and Development, 7(4), 259-270. DOI: https://doi.org/10.1046/j.1360-3736.2003.00185.x [GS Search]

McCarthy, J., Minsky, M., Rochester, N. \& Shannon, C. (2006). A Proposal for the Dartmouth Summer Research Project on Artificial Intelligence, August 31, 1955. AI Magazine, 27(4), 12. DOI: https://doi.org/10.1609/aimag.v27i4.1904 [GS Search]

Mora, N., Caballé, S. \& Daradoumis, T. (2016). Providing a multi-fold assessment framework to virtualized collaborative learning in support for engineering education. International Journal of Emerging Technologies in Learning (iJET), 11(7), 41-51. DOI: https://doi.org/10.3991/ijet.v11i07.5882 [GS Search]

Moodle (2018). About Moodle. Recuperado em 17 dezembro, 2018, de https://docs.moodle.org/36/en/About_Moodle/

Nilsson, N. (2014). Principles of artificial intelligence. Massachusetts: Morgan Kaufmann. DOI: https://doi.org/10.1016/C2009-0-27546-5 [GS Search]

Njenga, S., Oboko, R., Omwenga, E. \& Maina, E. (2017). Use of Intelligent Agents in Collaborative M-Learning: Case of Facilitating Group Learner Interactions. International Journal of Modern Education and Computer Science, 9(10), 18. DOI: https://doi.org/10.5815/ijmecs.2017.10.03 [GS Search] 
Noroozi, O., Weinberger, A., Biemans, H. J., Mulder, M. \& Chizari, M. (2012). Argumentationbased computer supported collaborative learning (ABCSCL): A synthesis of 15 years of research. Educational Research Review, 7(2), 79-106. DOI: https://doi.org/10.1016/j.edurev.2011.11.006 [GS Search]

Nwana, H. (1990). Intelligent tutoring systems: an overview. Artificial Intelligence Review, 4(4), 251-277. DOI: https://doi.org/10.1007/bf00168958 [GS Search]

O’Leary, R. \& Ramsden, A. (2002). Virtual learning environments. In The Handbook for Economics Lecturers: A Set of Guides to Inform and Improve the Practices of University Teachers of Economics. Bristol, UK: Learning and Teaching Support Network Generic Centre/ALT Guides, LTSN. [GS Search]

Patriarcheas, K. \& Xenos, M. (2010). Educational Techniques Comparative Study by Using Combined Environment via Computer and Mobile Devices in Asynchronous Discussion Forum. 2010 Ninth International Conference on Mobile Business and 2010 Ninth Global Mobility Roundtable (ICMB-GMR), Athens (pp. 297-304). DOI: https://doi.org/10.1109/ICMB-GMR.2010.25 [GS Search]

phpBB Limited (2014). About phpBB. Recuperado em 17 dezembro, 2018, de https://www.phpbb.com/about/?sid=4863513069010f391c626a5526522f05

Ramaiah, C. (1995). Bulletin board systems for libraries. DESIDOC Bulletin of Information Technology, 15(4), 23-31. DOI: https://doi.org/10.14429/djlit.15.4.3191 [GS Search]

Russell, S. \& Norvig, P. (1995). Artificial Intelligence: A Modern Approach. New Jersey: Prentice Hall. [GS Search]

Salihoun, M., Guerouate, F., Berbiche, N. \& Sbihi, M. (2017). How to Assist Tutors to Rebuild Groups Within an ITS by Exploiting Traces. Case of a Closed Forum. International Journal of Emerging Technologies in Learning (iJET), 12(3), 169-181. DOI: https://doi.org/10.3991/ijet.v12i03.6506 [GS Search]

Saqr, M., Fors, U., Tedre, M. \& Nouri, J. (2018). How social network analysis can be used to monitor online collaborative learning and guide an informed intervention. PloSone, 13(3). DOI: https://doi.org/10.1371/journal.pone.0194777 [GS Search]

Siebra, S. \& Machiavelli, J. (2015). Introdução à Educação a Distância e ao Ambiente Virtual de Aprendizagem. Recife: Editora Universitária da UFPE, 108p. [GS Search]

Silva, J., Ramos, L. \& Noronha, D. (2006). Base de dados. In Población, D.,Witter, G.,Silva,. Comunicação e produção científica: contexto, indicadores, avaliação (pp. 263-285). São Paulo: Angellara. [GS Search]

Troussas, C., Virvou, M. \& Alepis, E. (2014). Collaborative learning: Group interaction in an intelligent mobile-assisted multiple language learning system. Informatics in Education, 13(2), 279-292. DOI: https://doi.org/10.15388/infedu.2014.08 [GS Search]

Van Weijen, D. (2012). The Language of (Future) Scientific Communication. Recuperado em 06 dezembro, 2018, de https://www.researchtrends.com/issue-31-november-2012/thelanguage-of-future-scientific-communication [GS Search]

Vassileva, J., McCalla, G. \& Greer, J. (2016). From Small Seeds Grow Fruitful Trees: How the PHelpS Peer Help System Stimulated a Diverse and Innovative Research Agenda over 15 Years. International Journal of Artificial Intelligence in Education, (26)1, 431-447. DOI: https://doi.org/10.1007/s40593-015-0073-9 [GS Search] 
Webb, E., Jones, A., Barker, P. \& van Schaik, P. (2004). Using e-learning dialogues in higher education. Innovations in Education and Teaching International, 41(1), 93 - 103. DOI: https://doi.org/10.1080/1470329032000172748 [GS Search]

Wise, A., Hausknecht, S., \& Zhao, Y. (2014). Attending to others' posts in asynchronous discussions: Learners' online "listening” and its relationship to speaking. International Journal of Computer-Supported Collaborative Learning, 9(2), 185-209. DOI: https://doi.org/10.1007/s11412-014-9192-9 [GS Search]

Zaidi, A., Shane, B. \& Hannah, A. (2019). Review of the online learning and artificial intelligence education market. DfE research report no. DFE-00112-2019. Manchester Department for Education. Recuperado em 26 julho, 2019, de https://www.gov.uk/government/publications/review-of-the-online-learning-and-artificialintelligence-education-market [GS Search] 
Apêndice- Síntese de informações dos artigos selecionados

\begin{tabular}{|c|c|c|c|c|c|}
\hline Artigo & Autores & País & \begin{tabular}{|l|}
$\begin{array}{l}\text { Ambiente de } \\
\text { aplicação }\end{array}$ \\
\end{tabular} & Nível de ensino & Técnica IA \\
\hline $\begin{array}{l}\text { Using machine learning } \\
\text { techniques to support group } \\
\text { formation in an online } \\
\text { collaborative learning } \\
\text { environment }\end{array}$ & $\begin{array}{l}\text { Maina, E., } \\
\text { Oboko, R., } \\
\text { Waiganjo, P. } \\
(2017)\end{array}$ & Quênia & AVA (Moodle) & $\begin{array}{l}\text { Classe com } 36 \\
\text { alunos } \\
\text { (instituição não } \\
\text { especificada) }\end{array}$ & $\begin{array}{l}\text { - Aprendizado de máquina } \\
\text { - k-médias } \\
\text { - Expectation maximization (EM) }\end{array}$ \\
\hline $\begin{array}{l}\text { Use of Intelligent Agents in } \\
\text { Collaborative M-Learning: } \\
\text { Case of Facilitating Group } \\
\text { Learner Interactions }\end{array}$ & $\begin{array}{l}\text { Njenga, S., } \\
\text { Oboko, R., } \\
\text { Omwenga, } \\
\text { E., Maina, E. } \\
\text { (2017) } \\
\end{array}$ & Quênia & AVA (Moodle) & $\begin{array}{l}\text { Universidade } \\
\text { local no Quênia } \\
\text { (não } \\
\text { especificada) }\end{array}$ & - Agentes inteligentes \\
\hline $\begin{array}{l}\text { Providing a multi-fold } \\
\text { assessment framework to } \\
\text { virtualized collaborative } \\
\text { learning in support for } \\
\text { engineering education }\end{array}$ & $\begin{array}{l}\text { Mora, N. ; } \\
\text { Caballé, S. ; } \\
\text { Daradoumis, } \\
\text { T. (2016) }\end{array}$ & Espanha & \begin{tabular}{|l} 
- AVA (Moodle e \\
Virtual Campus) \\
- Sistema próprio de \\
visualização \\
(Virtualized \\
collaborative session \\
- VCS) \\
\end{tabular} & $\begin{array}{l}\text { Open University } \\
\text { of Catalonia } \\
\text { (UOC) e Cadiz } \\
\text { University } \\
\text { (UCA) }\end{array}$ & $\begin{array}{l}\text { - Regras baseadas em lógica fuzzy } \\
\text { - Processamento de linguagem } \\
\text { natural }\end{array}$ \\
\hline $\begin{array}{l}\text { How to assist tutors to } \\
\text { rebuild groups within an ITS } \\
\text { by exploiting traces. Case of } \\
\text { a closed forum }\end{array}$ & $\begin{array}{l}\text { Salihoun, M. } \\
\text {; Guerouate, } \\
\text { F. ; Berbiche, } \\
\text { N. ; Sbihi, M } \\
\text { (2017). } \\
\end{array}$ & Marrocos & AVA (Moodle) & $\begin{array}{l}\text { Mohammed V } \\
\text { University in } \\
\text { Rabat }\end{array}$ & - Algoritmo de busca local 2-opt \\
\hline $\begin{array}{l}\text { Experiences at the Open } \\
\text { University of Catalonia with } \\
\text { the virtualization of live } \\
\text { collaborative learning in } \\
\text { support for engineering } \\
\text { education }\end{array}$ & $\begin{array}{l}\text { Caballé, S., } \\
\text { Gañán, D., } \\
\text { Gómez, M., } \\
\text { Conesa, J., } \\
\text { Rius, A., } \\
\text { Santanach, F. } \\
\text { (2014) }\end{array}$ & Espanha & $\begin{array}{l}\text { - Web forum baseado } \\
\text { em phpBB } \\
\text { - Sistema próprio de } \\
\text { visualização } \\
\text { (virtualized } \\
\text { collaborative session } \\
\text { - VCS) }\end{array}$ & $\begin{array}{l}\text { Open University } \\
\text { of Catalonia } \\
\text { (UOC) }\end{array}$ & $\begin{array}{l}\text { - Regras baseadas em lógica fuzzy } \\
\text { - Processamento de linguagem } \\
\text { natural }\end{array}$ \\
\hline \begin{tabular}{|l|} 
Developing a collaborative \\
MOOC learning environment \\
utilizing video sharing with \\
discussion summarization as \\
added-value \\
\end{tabular} & $\begin{array}{l}\text { Al-Mousa, } \\
\text { M. ; Fiaidhi, } \\
\text { J. (2014) }\end{array}$ & Canadá & AVA (Moodle) & $\begin{array}{l}\text { MOOC oferecido } \\
\text { por Universidade } \\
\text { (não } \\
\text { especificada) }\end{array}$ & $\begin{array}{l}\text { - Processamento de linguagem } \\
\text { natural } \\
\text { - Algoritmos de poda }\end{array}$ \\
\hline $\begin{array}{l}\text { Collaborative Learning: } \\
\text { Group Interaction in an } \\
\text { Intelligent Mobile-Assisted } \\
\text { Multiple Language Learning } \\
\text { System }\end{array}$ & $\begin{array}{l}\text { Troussas, C.; } \\
\text { Virvou, M.; } \\
\text { Alepis, E. } \\
\text { (2014) }\end{array}$ & Grécia & Sistema mobile & $\begin{array}{l}\text { Ensino de } \\
\text { idiomas }\end{array}$ & $\begin{array}{l}\text { - Processamento de linguagem } \\
\text { natural } \\
\text { - Classificação }\end{array}$ \\
\hline
\end{tabular}




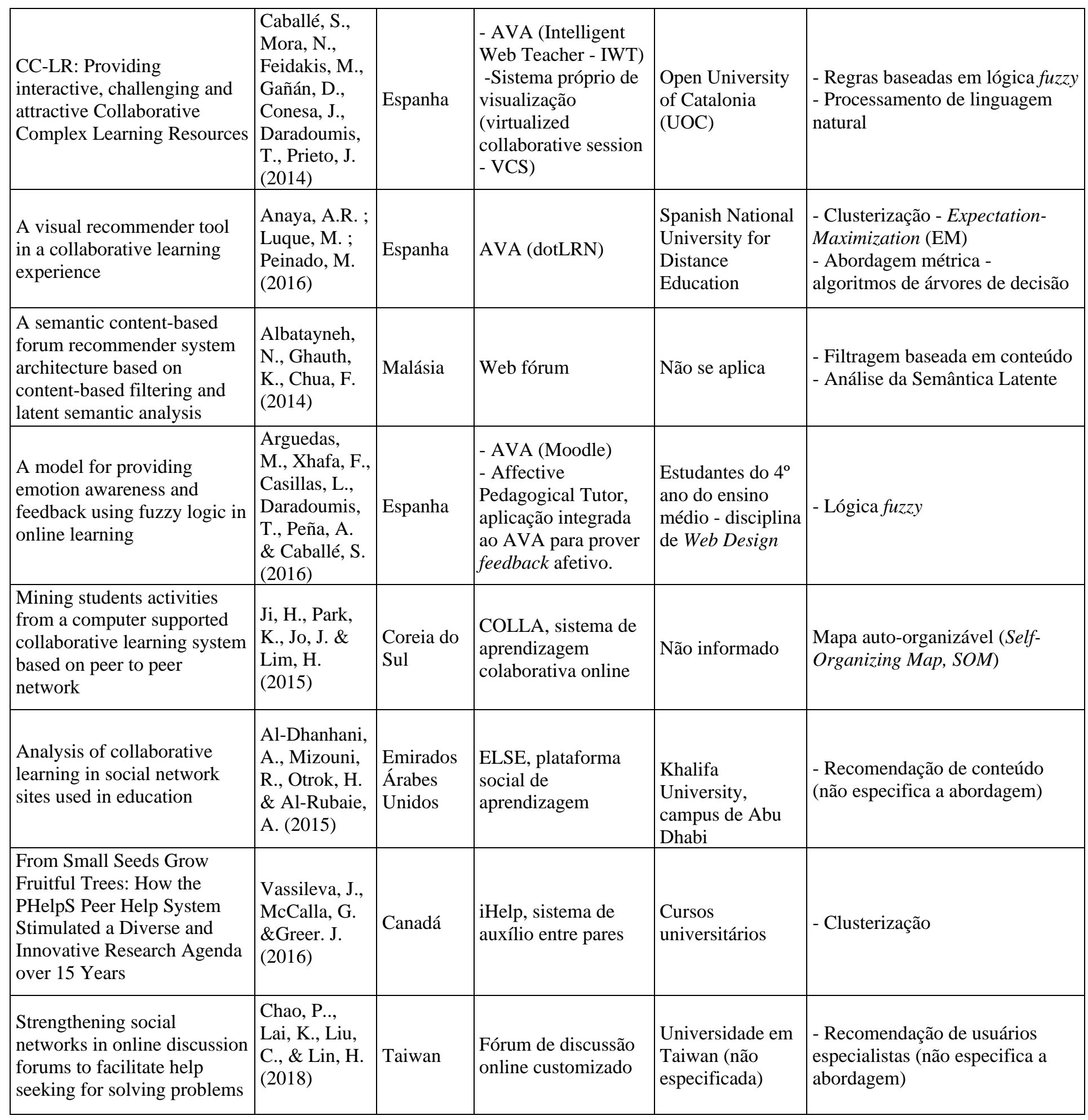

